\title{
GASTRIC ULCER AFTER SHORT-TERM NONSTEROIDAL ANTI-INFLAMMATORY DRUGS ADMINISTRATION IN A SMALL CHILD - CASE REPORT
}

\author{
Ancuta Ignat', Gabriela Paduraru', Angelica Cristina Marin', \\ Anamaria Ciubara ${ }^{2}$, Valeriu V. Lupu', Marin Burlea ${ }^{1}$ \\ ${ }^{1}$ Pediatrics Department, "Gr. T. Popa" University of Medicine and Pharmacy, Iasi \\ ${ }^{2}$ Psychiatry Department, "Gr. T. Popa” University of Medicine and Pharmacy, Iasi
}

\begin{abstract}
Nonsteroidal anti-inflammatory agents (NSAIDs) produce gastric lesions through two mechanisms: local irritation and systemic action. A 2 year and 10 months old female received NSAID for acute upper respiratory infection for 2 days and she developed coffee ground vomitus one day later. Upper gastrointestinal endoscopy revealed a gastric ulcer. She was negative for $H$. pylori infection, and she was diagnosed with NSAID - induced acute gastric ulcer in the absence of other causes of gastric ulcer. Gastric ulcers develop very rarely after a short-term administration of NSAIDs, which prompted us to report this case. Balancing the risk and the benefit of eradication therapy, it is indicated, for every patient who must follow a chronic treatment with anti-inflammatory drugs, to look for possible associated risk factors.
\end{abstract}

Keywords: anti-inflammatory drugs (NSAIDs), gastric ulcer, child

\section{INTRODUCTION}

Gastrointestinal hemorrhage is one of the most serious problems which have to be diagnosed and treated by the pediatric gastroenterologist. Severe gastrointestinal bleedings are rare in the pediatric population and therefore, they are not very well documented. (1)

Gastric ulcer is a multifactorial disease and represents the limited loss of substance to the mucosa, submucosa and muscularis. It is the result of an imbalance between mucosal protective mechanisms and aggressive factors. (2)

Nonsteroidal anti-inflammatory agents (NSAIDs) are a heterogeneous group of often unrelated chemical substances acting on certain stages of the inflammatory response in order to reduce it. They are commonly used in pediatric practice in young children for fever or pain control. High doses or prolonged use are associated with numerous gastrointestinal complications. NSAIDs produce gastric lesions through two mechanisms: local irritation and systemic action. NSAID-induced gastric ulcers occur mainly in the gastric antrum and are characterized by being multiple in numbers, irregular in shape, deeply undermined or huge. However, deeply undermined or huge gastric ulcers usually develop during the long-term administration of NSAIDs. $(3,4)$

\section{CASE REPORT}

A 2 year and 10 months old female received NSAIDs for acute upper respiratory infection for 2 days, developed coffee ground vomitus and epigastric pain one day later, and was admitted to our hospital.

Anamnesis reports a respiratory disease for $48 \mathrm{~h}$ treated symptomatically at home with ibuprofen three doses of $150 \mathrm{mg} / 8 \mathrm{~h}$. The patient was not taking regular medication. From the history we discover that the patient didn't have any dyspeptic episode before despite of the fact that in the infancy 
she took isolated doses of ibuprofen. In her family there is no record of any members diagnosticated with gastroduodenal ulcer or Helicobacter pylori infection.

Physical examination revealed influenced general condition, pale and dehydrated skin, suffering face, pharyngeal congestion, and distended abdomen, sensitive to superficial and deep palpation with maximum intensity in the epigastrium. During hospitalization she had dyspeptic episodes accompanied by hematemesis and epigastric pain.

Laboratory investigations revealed minor hepatocytolisis syndrome, but coagulation tests and abdominal ultrasound were normal.

Upper digestive endoscopy highlights a $2 / 3 \mathrm{~cm}$ ovoid ulceration in the stomach at $2 \mathrm{~cm}$ from the pylorus, antral purpuric gastric mucosa, and fluid stasis with food scraps (Fig. 1). The rapid urease test was negative, and Helicobacter pylori was not found at histopathological examination.
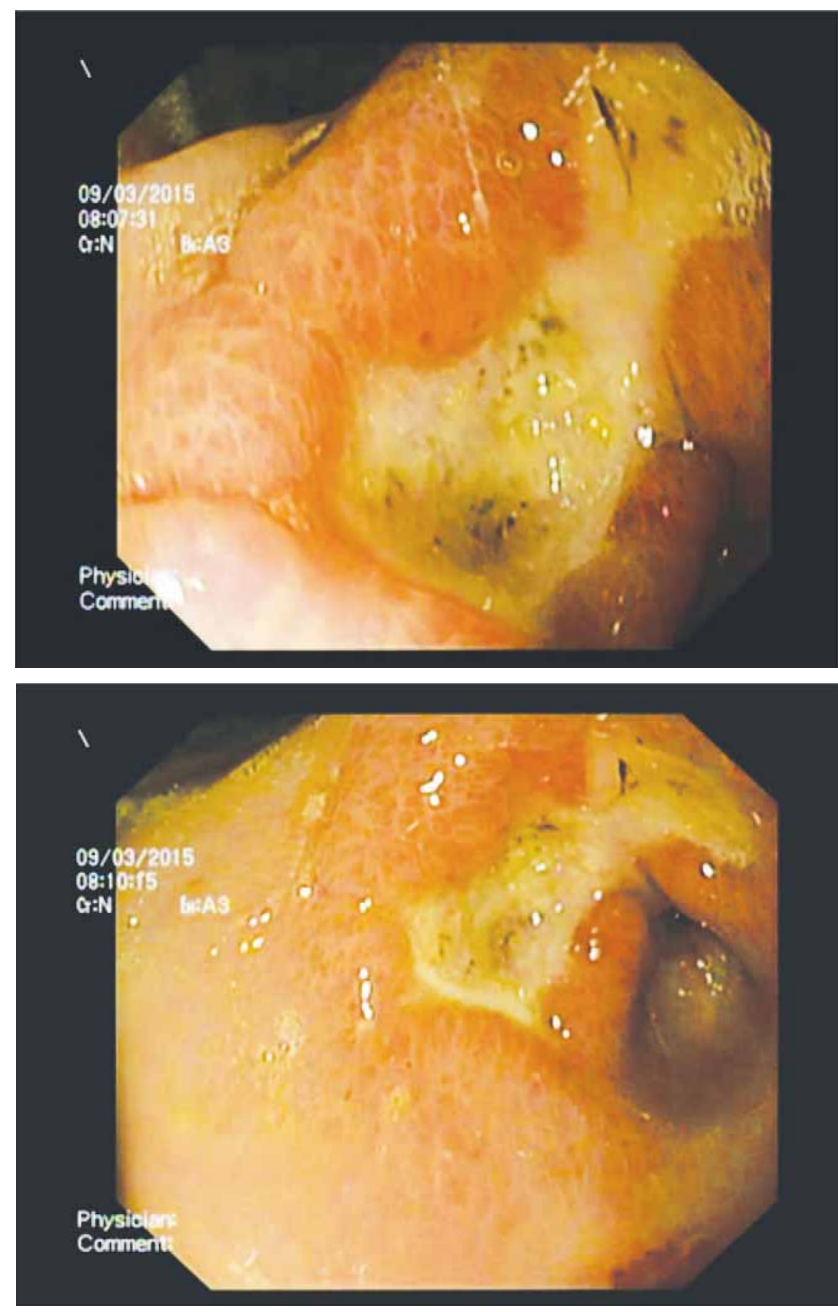

FIGURE 1. Upper digestive endoscopy showing a $2 / 3 \mathrm{~cm}$ ovoid prepiloric ulceration

The child received hemostatics and intravenous PPI, followed by oral PPI. The patient was dis- charged after 6 days with recommendation for oral PPI (Esomeprazole $1 \mathrm{mg} / \mathrm{kgc} /$ day) for six weeks. The evolution was favorable after the dietary and medical treatment.

The distinguishing feature of this case is the patient's small age and the precocious digestive complication developed at the use of a recommended doses of NSAIDs in the absence of any factors of risk.

\section{DISCUSSIONS}

The "coffee grounds" vomit shows a long contact of the blood with the gastric juice, which allows under the action of the chlorine hydride the transformation of hemoglobin into hematine hydrochloride. The vomit which contains red blood shows an active hemorrhage, which determines a short-term contact of the blood with the gastric juice.

Gastrointestinal (GI) bleeding in infants and children is a fairly common problem, accounting for $10-20 \%$ of referrals to pediatric gastroenterologists. However, it is usually limited in volume, allowing time for diagnosis and treatment. (1)

The etiology of upper gastrointestinal bleeding in children is definitely age related. Peptic ulceration, gastritis (due to stress or drugs), varices, epistaxis, Mallory-Weiss syndrome are the most common etiologies in children 3-5 years old. $(2,5)$

Drugs such as NSAIDs, steroids and antibiotics, which often generate mucosal damage, cause bleeding and the risk increases, if there is history of chronically usage of drugs, such as anticonvulsants or long-term oral antibiotics. Prolonged use of NSAIDs is associated with gastrointestinal disorders: gastritis, ulcers, gastrointestinal bleeding. (6)

In adults with chronic NSAIDs therapy, gastric ulcer is detected by endoscopy at a rate of $15-30 \%$. (7) In children with digestive symptoms, that are diagnosed with rheumatoid arthritis in chronic NSAIDs treatment, over $75 \%$ it presents antral lesions and ulcers. (8)

Two mechanisms are reportedly involved in acidic type NSAID-induced gastric mucosal injury in a synergistic manner: NSAIDs acquire the ability to permeate cell membranes in an acidic environment and accumulate in epithelial cells, resulting in metabolic disturbances, leading to cellular disruption and NSAIDs inhibit cyclooxygenases, resulting in a decrease in the production of endogenous prostaglandin (PG), as the major protective factor in the gastric mucosa, leading to mucosal injury. (9) 
Comparative studies in pediatric patients treated with acetaminophen/NSAIDs concluded that gastrointestinal bleeding appears only in patients with NSAIDs. The factors associated with NSAIDs use in the determination of gastrointestinal complications, are: pre-existing ulcers, multiple or high doses of NSAIDs, co-morbidities (rheumatoid arthritis), corticosteroids or anticoagulants, infection with Helicobacter pylori. $(2,10)$

It has been noted that a prodrug NSAID also reduces PG production, and the reflux of its active component (excreted from the liver into the bile) from the duodenum into the stomach is involved in the mechanism of gastric mucosal injury. NSAIDinduced gastric mucosal injury may occur at any point after the start of administration. (11)

Clinical data, but especially the increasing quality and accuracy of laboratory diagnosis, lead to a

\section{REFERENCES}

1. Pant C., Sankararaman S., Deshpande A., Olyaee M., Anderson M.P., Sferra T.J. Gastrointestinal bleeding in hospitalized children in the United States. Curr Med Res Opin. 2014 Jun. 30(6):1065-9

2. Kalyoncu D., Urganci N., Cetinkaya F. Etiology of upper gastrointestinal bleeding in young children. Indian J Pediatr. 2009 Sep. 76(9):899-901

3. Vaquero Sosa E., Bodas Pinedo A., Maluenda Carrillo C. Gastrointestinal bleeding following ingestion of low-dose ibuprofen. An Pediatr (Barc). 2012 Jun 18

4. Fox V.L. Gastrointestinal bleeding in infancy and childhood. Gastroenterol Clin North Am 2000; 29:37-66

5. Straube S., Tramer M.R., Moore R.A., Derry S., McQuay H.J. Mortality with upper gastrointestinal bleeding and perforation: effects of time and NSAID use. BMC Gastroenterol. 2009 Jun 5. 9:41

6. Reveiz L., Guerrero-Lozano R., Camacho A., Yara L., Mosquera P.A. Stress ulcer, gastritis, and gastrointestinal bleeding prophylaxis in critically ill pediatric patients: a systematic review. Pediatr Crit Care Med. 2010 Jan. 11(1):124-32 low rate of complications of these diseases, by applying multimodal treatment strategies and improving constantly.

\section{CONCLUSIONS}

Pediatric patients younger than 36 months are at increased risk of developing gastrointestinal bleeding, after administration of the first dose of NSAIDs.

Acetaminophen is safer, when it is used as a first choice medication for fever control in children.

The individual particularities of NSAIDs metabolism, depending the age and/or the enzymes can encourage the appearance of digestive complications with early start at doses adequate to the age.

7. Berezin S.H., Bostwick H.E., Halata M.S., Feerick J., Newman L.J., Medow M.S. Gastrointestinal bleeding in children following ingestion of low-dose ibuprofen. J Pediatr Gastroenterol Nutr, 44(4), 506-8, 2007

8. Owensby S., Taylor K., Wilkins T. Diagnosis and management of upper gastrointestinal bleeding in children. J Am Board Fam Med. 2015 Jan-Feb. 28 (1):134-45

9. Laine L., Takeuchi K., Tarnawski A. Gastric mucosal defense and cytoprotection: bench to bedside. Gastroenterology. 2008; 135:41-60

10. McCarty D.M. Nonsteroidal anti-inflammatory drug-induced ulcers: management by traditional therapies. Gastroenterology 1989; 96:662-674

11. Laine L., Curtis S.P., Cryer B., Kaur A., Cannon C.P. Risk factors for NSAID-associated upper GI clinical events in a long-term prospective study of 34701 arthritis patients. Aliment Pharmacol Ther. 2010 Nov. 32(10):1240-8 